\title{
Optimal Capacitive Load Matching of Micro Electret Power Generators
}

\author{
W. Li, H. W. Lo, and Y. C. Tai \\ Department of Electrical Engineering, California Institute of Technology, $1200 \mathrm{E}$ \\ California Blvd, Pasadena, California 91125, USA \\ This paper presents a model of micro-electret power generators. \\ This model uses two capacitors with fixed charge density and \\ variable area to model the actual micro electret power generator. \\ Simulations of power output with capacitive loads and resistive \\ loads are presented. The power output decreases as the load \\ capacitance increases while it increases as the resistive load \\ increases. To verify the model and simulation results, power output \\ generation experiments are performed and the results confirm the \\ simulation. To collect useful energy from the power generator, a \\ small capacitive load, rather than a resistive load, is required.
}

\section{Introduction}

During the last decade, research on energy harvesting has drawn much attention. "Energy Harvesting" is the term used to describe converting wasted ambient energy into useable electrical energy. And many approaches of the micro power generators have been demonstrated, such as the electromagnetic paradigm (2-3), the piezoelectric paradigm (4-5) and the electret paradigm (6).

The micro electromagnetic generator also uses the same principles of large-scale generators. A linear electromagnetic power generator was presented at the PowerMEMS 2004 conference (7), which is fabricated by using the micromachined processes. The size of this packaged 2-terminal version power generator is about 30 cubic centimeters, weighs 50 grams, and can deliver about $4 \mathrm{~mW}$ at $100 \mathrm{~Hz}$ vibration frequency with an acceleration of $0.4 \mathrm{~g}$.

The most aggressive development in mechanical energy harvesting devices has used piezoelectric materials. These kinds of materials can convert mechanical stress to an electrical polarization, which can then induce a current in an external circuit. The piezoelectric material used is typically lead zirconate titanate (PBT) with a perovskite crystalline lattice. Any piezoelectric material, such as porous electrets or lead barium titanate also can be used. The ability of the material to convert mechanical force to electrical energy is limited by the efficiency of the piezoelectric material to convert force to charge. The performances of these devices are limited to a narrow bandwidth about the mechanical resonant frequency due to their spring-proof-mass design. Furthermore, this problem also limited the application area for those energy harvesting devices.

In 2003, Boland et al demonstrated the first liquid-rotor Teflon AF electrets power generator by using the displacement current mechanism (8). His design overcomes the resonant frequency problem and gives a broad band operation. A power output of $0.43 \mu \mathrm{W}$ with a $28 \mathrm{~Hz}$ shaker has been reported. Another type of electret power generator with CYTOP film has been shown by Tsutsumino et al (9). Their linear solid-rotor 
power generator can produce $38 \mathrm{uW}$ with a $20 \mathrm{~Hz}$ shaker. But these types of electret power generators have a big issue with their gap control between the rotor and stator. A very careful gap control is required to reach a good performance. A new electret power generator with simple packaging and requires no precise separation gap control is presented by Lo (1).

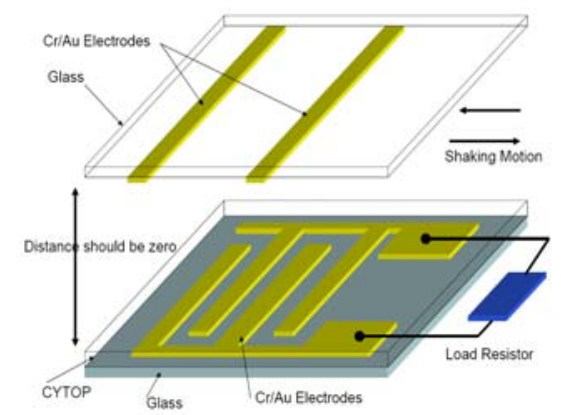

Figure 1. Schematic of the electrets power generator presented by Lo (1).

This electret power generator is fabricated using micromachining techniques to provide patterned electrodes on two glass substrates. The bottom "stator" plate has an interdigital metal electrode design covered by CYTOP electret. The top "rotor" plate is simply a piece of glass with floating metal strips and $1 / 2$ of the spatial frequency of the stator plate. In seismic operation, the amount of the image charge on the stator electrodes is charged due to the change of the overlapping area of two metal electrodes, producing electric current in the external circuit.

Table I shows the power densities of several kinds of power generators. As we can see, energy can be captured from the environment and does not need to be stored in a chemical form. And the stored energy is insufficient for devices that are designed to operate independently over long period of time. Also, the electrostatic and piezoelectric energy harvesters cannot provide a high energy density. As a result, the electrets power generation which can provide a much higher power density could be an interesting research field with bright future.

TABLE I. Power densities of various electrical power generators, Flux density measured in $\left(\mu \mathrm{W} / \mathrm{cm}^{2}\right)$. (10)

\begin{tabular}{lcc}
\hline Power Density $\left(\boldsymbol{\mu W} / \mathbf{c m}^{\mathbf{3}}\right)$ & Over one year & Over 10 years \\
\hline Solar (indoor) & 6 & 6 \\
Vibration (piezoelectric) & 100 & 100 \\
Vibration (Electrostatic) & 50 & 50 \\
Vibration (Electret) & 1000 & 1000 \\
Batteries (Lithium) & 45 & 4.5 \\
Fuel Cells (Methanol, theoretical) & 280 & 28 \\
\hline
\end{tabular}

\section{Micro Electret Power Generator Modeling}

We can consider the micro electret power generator as a constant charged capacitor. If we are able to change the capacitance, we can change the voltage across the capacitor and hence change the electric field. As a result, a displacement current can be generated. There are three ways to change the capacitance in the power generator: change the distance between the plates $d(t)$, change the overlap area of the capacitor $A(t)$ or change 
the permittivity of the capacitor $\varepsilon(\mathrm{t})$. In here, we model the power generator system with a circuit of fixed-charge variable area capacitors.
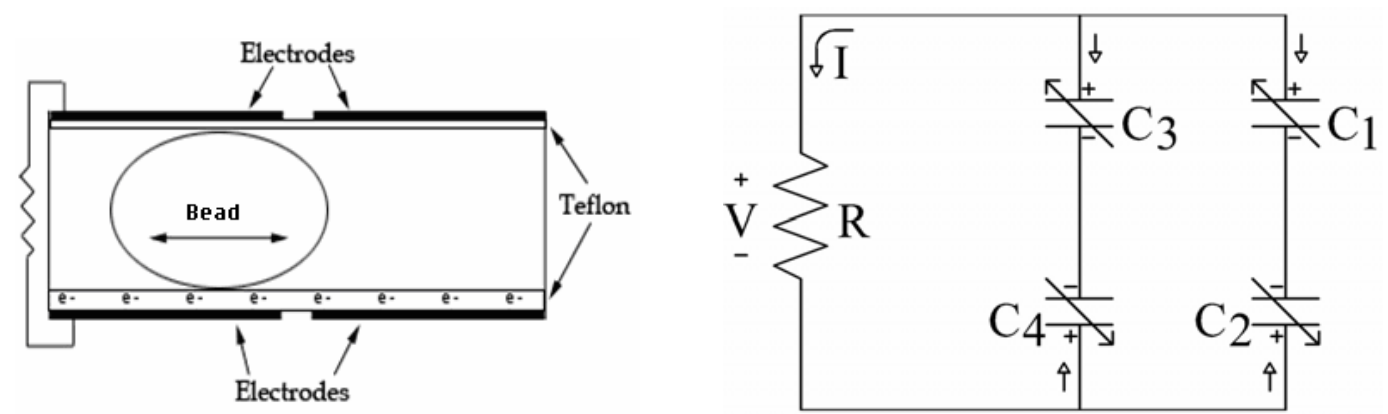

Figure 2. Left is the conceptual micro power generator image, right is the equivalent circuit for the power generator cell. (6)

We assume a simple capacitive structure and define

$$
\begin{aligned}
& C_{1}(t)=\frac{\varepsilon_{\text {metal }} \cdot A \cdot x(t)}{G} \\
& C_{2}(t)=\frac{\varepsilon_{\text {teffon }} \cdot A \cdot x(t)}{D} \\
& C_{3}(t)=\frac{\varepsilon_{0} \cdot A \cdot(1-x(t))}{G} \\
& C_{4}(t)=\frac{\varepsilon_{\text {teffon }} \cdot A \cdot(1-x(t))}{D}
\end{aligned}
$$

Also, we assume the shaking motion is sinusoidal $x(t)=\cos (\omega t)$. By using Kirchhoff's Voltage Law, we have:

$$
V=\frac{Q_{1}}{C_{1}}-\frac{Q_{2}}{C_{2}}=\frac{Q_{3}}{C_{3}}-\frac{Q_{4}}{C_{4}}
$$

$Q_{1}, Q_{2}, Q_{3}$ and $Q_{4}$ correspond to the charge stored on respective capacitors. With implanted charge $\rho$ and capacitor area $\mathrm{A}$, charge conservation gives us:

$$
\begin{aligned}
& Q_{1}+Q_{2}=\rho \cdot A \cdot x(t) \\
& Q_{3}+Q_{4}=\rho \cdot A \cdot(1-x(t))
\end{aligned}
$$




\section{Loading Optimization}

\section{$\underline{\text { Resistive Loading }}$}

In order to deliver the maximum power from the source to the load, a matching network is needed. At first, the pure resistive load has been analyzed. Look back to figure 2, if a resistive load is used, by the Kirchoff's Current Law:

$$
-\frac{V}{R}+\frac{d Q_{2}}{d t}+\frac{d Q_{4}}{d t}=0
$$

When we were trying to solve this equation analytically, we found out that this liner firstorder differential equation does not have a useful closed-form solution. However, it can be solved numerically using built-in ODE solvers in MatLab. By setting the values for all the parameters, we can numerically solve for the voltage and then find the power generated by

$$
P=\frac{V^{2}}{R}
$$

Figure 3 shows the output voltage versus time. We can see that the output voltage is also sinusoidal. With a $13 \mathrm{M} \Omega$ load, the peak-to-peak output voltage is about 18 volt.

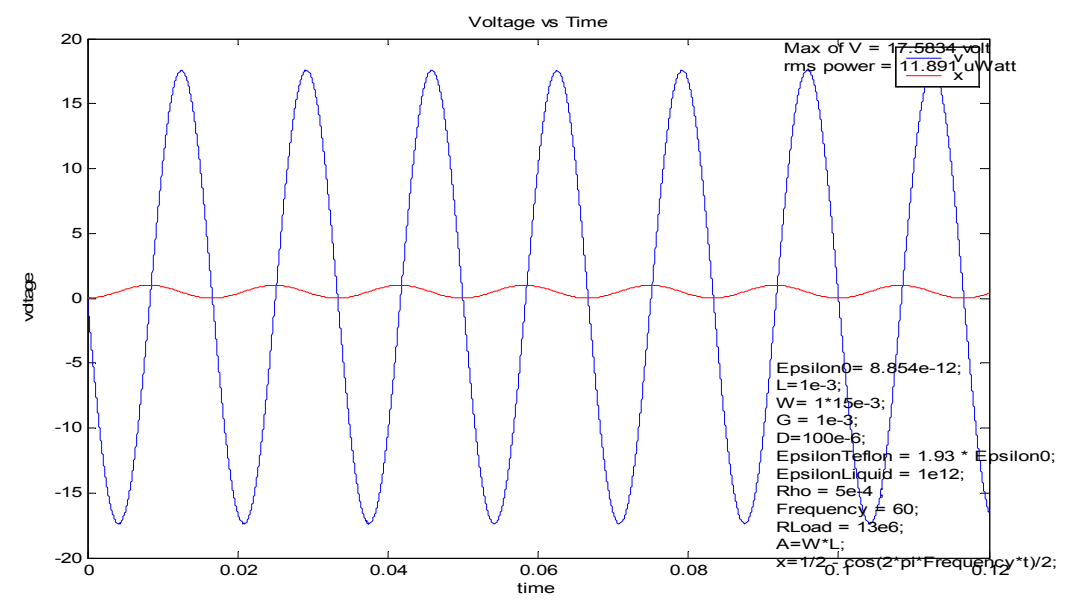

Figure 3. Output voltage versus time from the MatLab simulation with a $13 \mathrm{M} \Omega$ resistive load.

Figure 4 shows the power output versus frequency and load resistance. Also, we can see that the output power increases with the load resistance increases.

Since the electret power generator was modeled as a fixed-charge variable-area capacitor, with the resistive load, the maximum power output should occur when the time constant of the system matches the shaking frequency. In this case, the matching resistance is dependent of the shaking frequency. Furthermore, very large resistance (as we can se, $20 \mathrm{M} \Omega$ is still not big enough) is selected for the matching due to the small 
capacitance in the generator. This limits the performance of the power generators because the environment cannot provide a constant vibration frequency and a large resistance is not practical to make under the integrated circuit design limitation. As a result, a capacitive matching mechanism which is independent of the shaking frequency was explored.

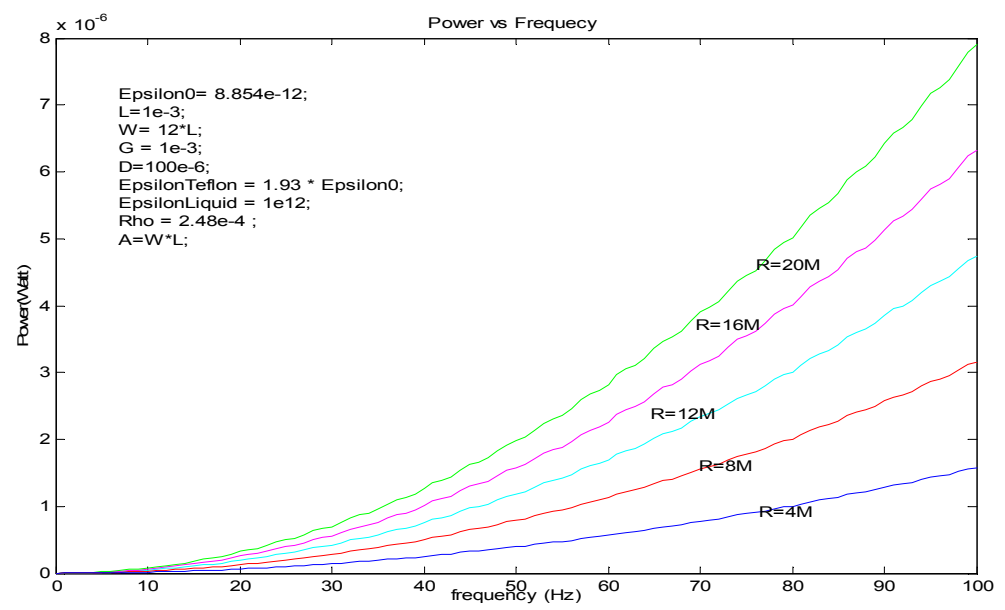

Figure 4. Output power versus frequency from the MatLab simulation.

\section{Capacitive Loading}

First, an analytical model for the capacitive loading micro electret power generator was developed.

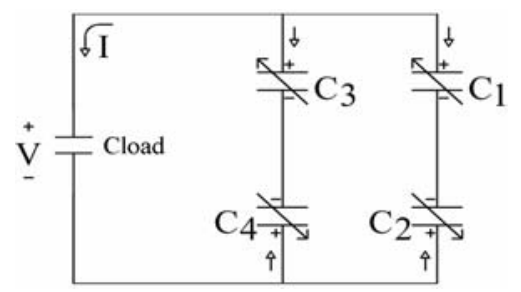

Figure 5. Equivalent circuit for the capacitive loading micro electret power generator.

Recall our previous micro power generator model; we solve equations [6] and [7] for charge Q:

$$
\begin{aligned}
Q_{2}= & \frac{-V+\rho A \alpha(t) \frac{1}{C_{1}}}{\frac{1}{C_{1}}+\frac{1}{C_{2}}} \\
Q_{4} & =\frac{-V+\rho A(1-\alpha(t)) \frac{1}{C_{3}}}{\frac{1}{C_{3}}+\frac{1}{C_{4}}}
\end{aligned}
$$


Then by Kirchhoff's Current Law, we have:

$$
\frac{\partial Q_{2}}{\partial t}+\frac{\partial Q_{4}}{\partial t}=C \cdot \frac{\partial V}{\partial t}
$$

We use Mathematica to solve this linear ODE and plot the voltage output. From the figure 6 , we can see that the output voltage for a capacitive load is also sinusoidal. For a luf capacitor load, the output peak to peak voltage is about $11 \mathrm{~V}$.

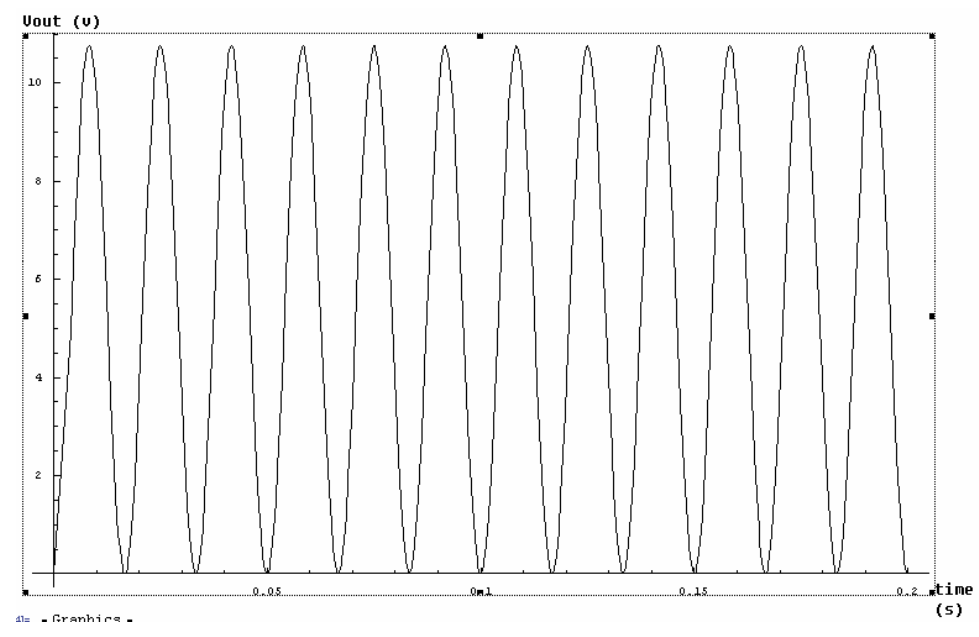

Figure 6. Mathematica plot for the output voltage versus time for a 1uf capacitor load.

From the power output plot in figure 7, we can see that the output power increases exponentially as the load capacitance decreases. The output power reaches its maximum when the load capacitance is matched, which is in nanofarad range due to out power generator design. So if we want to optimize our output power, we should use a smaller capacitor to storage charge. Also, we found out that using capacitor as a load can have a better power output from the power generator. For 5 uf capacitor load, at $60 \mathrm{~Hz}$ shaking frequency, the output power is $5 \mathrm{~mW}$ for 100 cells. So the output power for each cell is $50 \mathrm{uW}$. But according to figure 4 , for a $20 \mathrm{M}$ ohm resistor load, the output power at $60 \mathrm{~Hz}$ is only less than $3 \mathrm{uW}$. As a result, using a suitable capacitor as load is preferable.

In addition, our experimental data in figure 8 also shows a good agreement with our simulation. While the load capacitance approaching to the match capacitance of the generator, the output power increases.

As we can see from the simulation and experimental data, in order to get a high power output, a very small capacitor needs to be used to match with our power generator. But if the capacitor small, the energy stored in the capacitor is also very small, which is not enough to power other application devices. But if a large capacitor is used to store the energy, the efficiency of out micro power generator will be reduce significantly. As a result, this is a trade-off; we can just choose the smallest capacitor which can store enough energy to power our application. 


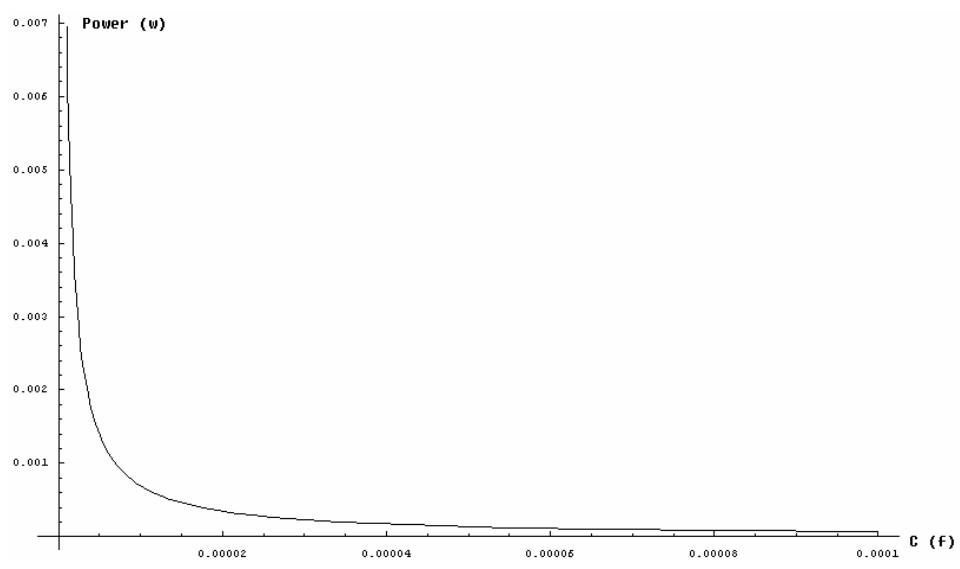

Figure 7. Mathematica plot for the output power versus pure capacitive load at $60 \mathrm{~Hz}$.

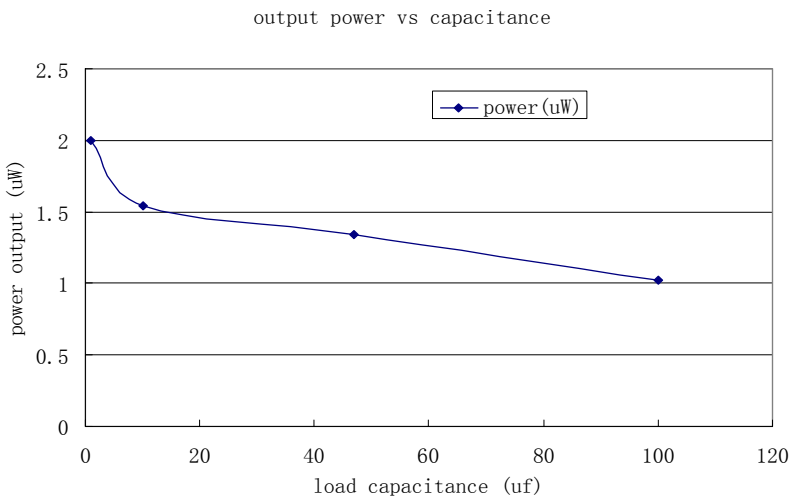

Figure 8. Experimental data for the output power versus load capacitance.

In addition, for a matching network, we understand that the load is better to be the conjugate of the generator output. Since we model the power generator as variable capacitor, in order to match well this generator, an inductive load should be used. But in the experiment, we found out that the low output current from our generator cannot drive an inductor. As a result, the inductive matching mechanism does not fit for out micro power generator.

\section{Conclusion}

Our capacitive matching mechanism can provide a higher power output than the conventional resistive matching. Also, the small capacitive load gives a higher flexibility for environment and is easier to implant in Integrated Circuits. For the future work, a switching capacitor circuit may be investigated. In that case, a small capacitor can be used to store the charge from the micro power generator, and then the switching circuit will accumulate this charge to a large storage capacitor for other applications. As a result, high output power can be reached by only loading the small capacitor to the power generator. 


\section{Acknowledgments}

The authors would like to thank Mr. Trevor Roper for his assistance with equipment and fabrication. We would also thank Tanya Owen, Christine Matsuki, Agnes Tong and other members of the Caltech Micromachining Laboratory for their assistance.

\section{References}

1. H.W. Lo, R.Whang, and Yu-Chong Tai, A Simplified Micro Electret Power Generator, Proc. Int. Conf. MEMS'07, (2007).

2. S.Roundy, P.K Wright, and K.S.J.Pister, Micro-electrostatic Vibration-toElectricity Converters, Proc.IMECE'02 (2002).

3. C.B. Williams, andR.B Yates, Analysis of a Micro-electric Generator for Microsystems, Sensors and Actuators, A, Vol.52, p. 8-11 (1996).

4. G.K Ottman, H.F.Hofmann, A.C. Bhatt, and G.A.Lesieutre, Adaptive Piezoelectric Energy Harvesting Circuit for Wireless Remote Power Supply, IEEE transaction on Power Electronics, Vol.17, No.5, (2002).

5. N.Shenck and J.A. Paradiso, Energy Scavenging with Shoe-mounted Piezoelectrics, IEEE micro, Vol.21, p. 30-42, (2001).

6. J. S. Boland, J.D.M. Messenger, H.W. Lo and Y.C. Tai, Arrayed Liquid Rotor Electret Power Generator Systems, Proc. Int. Conf. MEMS'05, (2005).

7. A. Huang, P.J. Chen, J. Boland, Y.C. Tai and C.M. Ho, Liquid-Rotor Electret Power Generator Array Energized by a MEMs-Based Pulsed Combustor, PowerMEMS04, (2004).

8. J. Boland, Y.H. Chao, Y. Suzuki and Y.C. Tai, Micro Electret Power Generator, Proc. Int. Conf. MEMS'03, (2003).

9. T. Tsutsumino, Y. Suzuki, N. Kasagi, and Y. Sakane, Seismic Power Generator Using High-performance Polymer Electret, Proc. Int. Conf. MEMS'06, (2006).

10. J. Boland, Thesis for the Degree of Doctor of Philosophy, p. 21, California Institute of Technology, (2005). 